\title{
CTA Atmospheric Calibration
}

\author{
Markus Gaug ${ }^{1,2, \star}$ \\ ${ }^{1}$ Unitat de Física de les Radiacions, Departament de Física, Universitat Autònoma de Barcelona, Spain \\ ${ }^{2}$ CERES-IEEC, Universitat Autònoma de Barcelona, Spain
}

\begin{abstract}
The main contribution to the systematic uncertainties of Atmospheric Imaging Cherenkov Telescopes (IACTs) currently stems from the uncertainty in the determination of atmospheric properties for a given moment of observation. This technique uses the atmosphere as a calorimeter of gamma-ray induced atmospheric air showers and measures the amount of Cherenkov light collected by large mirrors and focused towards a pixellized camera. Atmospheric conditions affect the measured Cherenkov light yield in several ways: the air-shower development itself, the variation of the Cherenkov angle with altitude and the loss of photons due to scattering and absorption of Cherenkov light. Although IACTs use to be located at astronomical sites, characterized by extremely clear atmospheric conditions, the local atmosphere should be continuously monitored, in terms of molecular density profiles, aerosol extinction profiles, and clouds. Moreover, a general understanding of the site and particularly aerosol climatology is desirable for a robust interpretation of the sensing instruments' data. Here, the strategy of the Cherenkov Telescope Array (CTA) on atmospheric monitoring is presented, designed to ensure that the related systematic uncertainties are brought down by at least a factor of two with respect to current installations. Additionally, a more intelligent scheduling scheme is aimed at, where source observations are selected using dedicated auxiliary atmospheric monitoring instruments and taking into account the feasibility of a later analysis according to the underlying scientific case. This plan should reduce data loss during offline data selection and enhance the effective duty cycle of the CTA.
\end{abstract}

\section{Introduction}

Over the past decade, Imaging Atmospheric Cherenkov Telescopes (IACTs) have opened a new observation window for gamma-ray astronomy: the Very High Energy (VHE) regime, ranging from tens of $\mathrm{GeV}$ to several tens of $\mathrm{TeV}$ gamma-ray energy. The technique being groundbased permits building large mirrors and exploit the huge area illuminated on ground by the Cherenkov light emitted by gamma-ray induced air showers. This makes it possible to achieve unprecedented sensitivities, orders of magnitude better than what can be achieved by satellites in that energy range [1]. So far, more than hundred VHE gammaray sources have been discovered by IACTs [2].

On the other hand, using the atmosphere as a calorimeter brings along a series of limitations on the accuracy with which the energy of an air shower can be reconstructed, and the exposure of the instrument calibrated. Albeit several efforts have been made in that direction $[3,4]$ and unlike other astronomical installations operating at lower energy ranges, there is still no direct cross-calibration with satellites available, let alone a test beam.

The main contribution to the systematic uncertainties of IACTs stems then from the uncertainty in the (heightand wavelength-dependent) atmospheric transmission for a given moment in time. Current estimates range around $15 \%$ for the accuracy with which the energy scale is cali-

\footnotetext{
^e-mail: markus.gaug@uab.cat
}

brated [5, 6] and a similar, or only little smaller number for exposure calibration. Both estimates are however based on selected data recorded during perfect atmospheric conditions and have to be considered lower limits for the general case of data taken under still acceptable conditions.

Atmospheric quality affects the measured Cherenkov yield in several ways [8]: the air-shower development itself depends on the molecular density profile along its trajectory as well as the variation of the Cherenkov angle. Further, the loss of Cherenkov photons is affected by absorbing molecules and, more importantly, scattering out of the camera field-of-view (FOV), both by molecular (Rayleigh) and aerosol (Mie) scattering. Clouds are usually thinner than the several kilometers long air showers and normally remove only a part of the emitted Cherenkov light from the FOV of an IACT. Since the mean interaction depth of the shower maximum depends on the primary gamma-ray energy, the altitude of a cloud layer can create energy dependent effects $[9,10]$. Also the case of negligible interaction of Cherenkov light with a (high) cloud is possible, if the shower energy is high enough and even for an optically thick cloud [11]. An altitude-dependent assessment of the atmospheric extinction is therefore mandatory, e.g. with the use of a LIDAR [12]. Multiple scattering of photons back into the camera in turn, has only a minor effect and can normally be neglected [8].

Despite several auxiliary instruments of IACT installations are currently used to measure atmospheric extinc- 
tion [13], or even the very same science data can be exploited to that aim to certain extent $[14,15]$, their outcome has been mainly used to retain good-quality observation time slots, and only a minor effort has been made to correct data with atmospheric information $[10,13,16]$. Only the MAGIC collaboration has started to routinely include simultaneously taken LIDAR measurements in its data analyses [12, 17-19].

Following the natural evolution of new astronomical instruments and observation windows, the next generation VHE gamma-ray observatory Cherenkov Telescope Array (CTA) will move from the discovery mission of its predecessors to perform population studies and precision measurements, resolving spectral features and the morphology of gamma-ray sources with unprecedented precision [20] ${ }^{1}$. A thorough calibration strategy for the used calorimeter, the air, must hence be included in its design, as well as ways to optimize its effective duty cycle, even after data selection and analysis cuts are applied [7]. Recently, the CTA site selection has been completed, with as outcome, for its Southern observatory (CTA-S), a plateau of the Cerro Armazones in Northern Chile, close to Paranal, while the "Roque de los Muchachos" Observatory (ORM) on the island of La Palma, Canary Islands, has been selected for its Northern part (CTA-N). Both observatories are located around $2200 \mathrm{~m}$ a.s.l. Knowledge of the concrete sites permits now to design tailored atmospheric calibration strategies for both.

This proceeding presents first several aspects of the atmosphere, necessary to understand CTA's atmospheric calibration strategy, followed by the designed calibration of the observed FOV. Later on, we will show how the effective duty cycle can be improved already during scheduling of sources.

\section{The atmosphere above CTA}

From the various atmospheric layers, only the troposphere (reaching from ground to $\sim 15 \mathrm{~km}$ a.s.l.), and sometimes parts of the tropopause are affected by variations of their optical properties. Only in the case of stratovolcanic eruptions, the lower stratosphere (15-20 km a.s.l.) may become also optically degraded [24]. Air molecules can travel to the top of the troposphere and back down in a few days, hence the mixing of the characteristics of the layer is fast [25]. The lowest part of the troposphere, enclosing most of the aerosol over-density, is called the "planetary boundary layer" (PBL), whose thickness increases during day and shrinks during night. Its top is characterized by a sharp decrease in particulates and condensates frequently leading to the creation of convective clouds. During night, the PBL may leave a residual layer on its top, the "nocturnal boundary layer" where pollutants emitted at the surface remain concentrated in a layer few tens of meters thick near the surface. At an astronomical site, this layer is expected at about one kilometer above ground,

\footnotetext{
${ }^{1}$ Several recent discoveries by current IACTs are already limited by the systematic uncertainties with which the instrument response is known. What was good enough for the discovery of sources, is now starting to limit performance [see e.g. 21-23].
}

however often showing an exponential decay of aerosol densities [26, 27].

While the molecular content of the atmosphere varies very slowly at a given location during the year, and slowly from place to place, aerosol concentrations can vary on time-scales of minutes and travel large, even intercontinental, distances [28]. Most of them are concentrated within the first $3 \mathrm{~km}$ of the troposphere, being the "free troposphere" above orders of magnitude cleaner. A "photometric night" at an astronomical site is practically identical with an undisturbed free troposphere, and a negligible amount of aerosols close to the ground. While groundlevel aerosols (due to the nocturnal boundary layer, but also from desert dust intrusions or biomass burning) typically degrade the transmission of all Cherenkov light [9], the vertical structure of higher altitude aerosols and clouds (including cirrus) must be determined since only a part (or even none!) of the emitted Cherenkov light suffers extinction. A correction is then more difficult, but nevertheless possible [17]. Stratospheric aerosols from volcanic eruption debris in turn, present for years after strong stratovolcanic eruptions, may cause a significant obscuration of the star light [24], but only influence the Cherenkov light from air showers of exceptionally high shower production heights and hence only at the very energy threshold of an IACT. Relying only on photometry of reference stars may introduce hence a strong bias difficult to correct for [8].

The understanding of the evolution of the main characteristics of aerosol (size, absorption and scattering of light, chemical composition, etc.) is extremely complex and a prime topic of current research, given their importance for the understanding of the world climate [29]. Light scattering and absorption by aerosols needs to be described by Mie theory or further developments of it, including nonsphericity of the scatterer [30]. Aerosols use to have larger refraction indices than the one of water, and typically show also a small imaginary part [31, 32]. Contrary to the typical $\lambda^{-4}$ wavelength dependency of Rayleigh-scattering molecules, aerosols show power-law indices (the so-called Ångström coefficients) from 0 to 1.5 , i.e. a much weaker dependency on wavelength $[33,35]$. Of special interest here is the ratio between extinction and backscatter coefficient, the "Lidar Ratio" (LR) which shows a strong dependency on relative humidity and can range from 15 to $80 \mathrm{sr}$ [33] for ground layer aerosols, and from 2 to $20 \mathrm{sr}$ in cirrus clouds [34] (the pure Rayleigh scattering case corresponds to $8 \pi / 3 \sim 8.4 \mathrm{sr}$ ). Elastic LIDARs need to make a-priori assumptions on this parameter, their main systematic uncertainty stems from the conversion of the (measured) backscatter coefficients to the (inferred) light extinction coefficients, as a function of height. Raman LIDARs in turn, are able to measure the LR directly.

\section{Overall Strategy}

Following the CTA's science and performance requirements, the allowed systematic uncertainty on the reconstructed overall energy scale of CTA permits some 7-8\% maximally as contribution from atmospheric calibration. This is in fact the uncertainty with which the Cherenkov 
photon density on ground is related to the air shower energy on average. The relation is estimated via comparison of the measured number of Cherenkov photons with its prediction from Monte Carlo (MC) simulations of gammaray induced showers. Although a given fixed atmosphere must be used for each simulation set, the CTA will aim for time-interval (TI)-wise simulations, configured each to match the given state of hardware and the respective atmospheric conditions.

Since Cherenkov photons can be emitted (and imaged) anywhere from about $20 \mathrm{~km}$ altitude to ground, the troposphere and lower stratosphere need to be characterized in a height-dependent way.

\subsection{The molecular profile}

A first topic of prime interest concerns the need (or not) to adapt molecular profiles for each simulation TI. Although initial work by Bernlöhr [8] pointed to principally possible large variations, of the order of up to $20 \%$ in Cherenkov light yield for mid-latitudes, the actual conditions at the locations of the H.E.S.S. and MAGIC telescopes [8, 36] resulted to be such that one average profile was sufficient as input for all MC simulations. The assumed error of only 2-3\% rms induced by this choice resulted to never limit their overall systematic uncertainty, by a factor of several times higher $[5,6]$. Whether such a statement is still true for the CTA, is currently being investigated [37, 38]. The difference is not only due to the stricter requirements on the CTA's accuracy, but also to its wider coverage of gamma-ray energies. Towards the high energy range (aiming at energies larger than $300 \mathrm{TeV}$ in the South) showers penetrate deeper into the atmosphere which in turn shows different density variations [see e.g. 37]. On the other side of the spectrum, very low energy showers develop in an around the tropopause where relative density variations, and their uncertainties, are largest [37].

Variations of the Cherenkov light yield are due to the differences in the height of the shower maximum, coupled with the height-dependent threshold for Cherenkov light emission [8, 39], and the modulation of the Cherenkov angle with the local density of air. If measurements or now-cast predictions with a precision of about $1 \mathrm{~g} / \mathrm{cm}^{2}$ at a height resolution of $20 \mathrm{~m}$ are available, about $2.5 \%$ uncertainty on the relative amount of radiation lengths of electrons and gamma rays can be achieved, and hence approximately a relative uncertainty on the shower energy of a same order of magnitude. Modern radio sondes can easily achieve such a precision, albeit diurnal and seasonal variations may be greater [27, 37]. Apart from air shower physics, the IACT measurement process is moreover determined by the production mechanism of Cherenkov light which itself depends on the refractive index (and hence more generally the density) of the surrounding air at its emission point. When the Cherenkov cone opening angle increases, the Cherenkov light gets spread over a larger area, decreasing the central light density. Bernlöhr [8] has shown that the central light density $\rho_{c}$ for vertical showers follows roughly:

$$
\rho_{c} \propto\left(h_{\mathrm{med}}^{*}-h_{\mathrm{obs}}\right)^{-2},
$$

where $h_{\text {med }}^{*}$ is the median height of emission for Cherenkov light near the core (typically 1000-1500 m below that of all Cherenkov light and hence the shower maximum) and $h_{\text {obs }}$ is the observatory altitude. From Eq. 1 follows immediately that any constant relative systematic offset of the molecular altitude profile is expected to create stronger relative deviations in Cherenkov light density for high shower energies (i.e. smaller values of $h_{\text {med }}^{*}$ ). Studies carried out for MAGIC, using the now-cast predictions of the "Global Data Assimilation System" (GDAS) ${ }^{2}$ for La Palma, have shown that a constant difference of $1 \%$ between the assumed and the true integrated density from ground to the shower maximum (corresponding to the rms of the GDAS predictions throughout one year) leads to differences in $\rho_{c}$ of about $0.5 \%, 1.5 \%, 3.5 \%$ and $6 \%$ for gamma-ray energies of $20 \mathrm{GeV}, 200 \mathrm{GeV}, 3 \mathrm{TeV}$ and $70 \mathrm{TeV}$, respectively applying Eq. 1. To these numbers, an additional contribution of the probable bias between the mean GDAS model and the true average annual atmosphere needs to be added, which must be validated by dedicated radio sonde campaigns [37], or a LIDAR sufficiently sensitive to the molecular profile [27], or from sophisticated analysis of high-resolution spectrometer data gathered by an optical telescope [40]. The CTA will carry out a radio sonde campaign at its Southern site and rely on a database covering several years of MAGIC LIDAR [12] data to assess the latter. A dedicated simulation effort is now ongoing in CTA [38] to directly assess the effect of typical and maximum variations of the density profile using the vast historical database of the GDAS ${ }^{3}$, together with assumptions on the accuracy of these. The outcome of these simulations should be ready for the next AtmoHEAD conference and answer the question of how many different molecular profiles will be required for the CTA data analysis.

Light absorbing molecules are of less importance for the CTA, given the drop of Cherenkov light intensity with increasing wavelength, and consequently the chosen sensitivity window of the telescope cameras, limited to between $290 \mathrm{~nm}$ and about $700 \mathrm{~nm}$. In this range, only few molecules (mainly $\mathrm{O}_{3}, \mathrm{NO}_{2}, \mathrm{H}_{2} \mathrm{O}$ and $\mathrm{O}_{2}$ ) absorb light at all, of which ozone creates the biggest effect, both in the Huggins band below $360 \mathrm{~nm}$ as well as the weak Chappuis band (375-650 nm). The strong Hartley band (220$310 \mathrm{~nm}$ ) reaches only marginally into the sensitive wavelength region of the CTA. The strongest systematic effect is expected from ozone intrusions into the upper troposphere, so-called "stratosphere-troposphere exchange" (STE) processes, best assessed by radio sondes with ozone measuring capabilities [41]. Since these come along with a considerably increased price adding to the already high cost of a thoroughly and systematically designed radio sonde campaign, cheaper methods have been investigated. For the North, and particularly the Canary Islands, Cuevas et al. have established an ozone climatology [42] using the "Potential Vorticity" (PV) parameter [43] obtained from "European Centre for Medium-Range Weather Forecasts"

\footnotetext{
${ }^{2}$ https://www.ncdc.noaa.gov/data-access/model-data/ model-datasets/global-data-assimilation-system-gdas, see also http: //earth.nullschool.net/

${ }^{3}$ or, alternatively the ECMWF http://www.ecmwf.int/
} 
$(\text { ECMWF })^{4}$ reanalysis, and compared with backward trajectories using the "Hybrid Single-Particle Lagrangian Integrated Trajectory" (HYSPLIT) ${ }^{5}$ code. They show that the Canary Islands are basically un-affected by STE processes throughout the year, a finding confirmed by S̆kerlak et al. [46]. However, the region of Mid-Northern Chile seems to be hot spot for STE [46], at least during the austral Summer months, centered about $10^{\circ}$ south of CTA-S. Rapid increases, on time scales of less than a day, in ozone mixing ratios linked with STE processes [44] have been observed at Cierro Tololo, a pristine mountain site about $5^{\circ}$ south of CTA-S. Strong deep stratospheric mass flux into the PBL was also observed by S̆kerlak et al. [46] during the austral winter months over the Andes, from $20-30^{\circ} \mathrm{S}$. An analysis of 20 years data of the same site [45] revealed an overall positive trend of $\sim 0.7 \mathrm{ppb} /$ decade in surface ozone, and strong seasonal trends, with maxima in October, again correlated with STE processes and during La Niña conditions. Generally, the contribution of ozone exchange with the stratosphere is predicted to increase with climate change [47]. Kimeswenger et al. [48] however suggested to build a specialized spectrograph mounted on an optical telescope to monitor the ozone profile. Whether ozone monitoring with a Cimel Sun photometer ${ }^{6}$ is feasible for the CTA-S still needs to be found out. The loss of Cherenkov light due to ozone is however expected to be less than $5 \%$, with an uncertainty of a few percent only.

\subsection{Aerosol characterization of the observed field-of-view by CTA}

The main contribution to systematic uncertainties in atmospheric calibration stems from aerosols and clouds which can show variability over minutes only. Consequently, aerosol extinction needs to be monitored on such time scales. Since some interpolation in time is always necessary and adds additional uncertainties, the combined precision and accuracy of each aerosol optical depth (AOD) measurement, as a function of emission altitude, should not exceed $2 \%$, with an altitude resolution of the order of one electron radiation length, i.e. $\sim 40 \mathrm{~g} / \mathrm{cm}^{2}$. Only the fine-structure of the nocturnal boundary layer need not be resolved [9]. Moreover, optically thick low clouds are of no interest since observations will be aborted anyhow under such conditions. Remain optically thin clouds, at any altitude, which are typically thinner or of the order of one radiation length. Hence most of the time, even the altitude resolved fine-structure of clouds are of no interest, only their integral optical depth.

The CTA has been designed to cover a wide FOV, reaching up to $10^{\circ}$ per telescope, and up to $20^{\circ}$ for the full array, if operated in divergent pointing mode. Such large FOVs entail the necessity to characterize a similarly large patch of the atmosphere, the characterization in only one line-of-sight results insufficient.

Raman LIDAR measurements are desirable to determine the altitude profile of aerosol extinction since

\footnotetext{
${ }^{4}$ www.ecmwf.int/

${ }^{5}$ www.arl.noaa.gov/HYSPLIT.php

${ }^{6}$ www.cimel.fr
}

they provide the required precision and accuracy for both ground-level aerosols (if equipped with near-range optics or if the LIDAR's system constant can be calibrated [see 27]) and the vertical structure of higher altitude aerosols and clouds.

Experience has shown that Raman LIDARs and their data analysis methods should be validated through recognized LIDAR networks, e.g. the EARLINET ${ }^{7}$ in Europe. We plan to install one Raman LIDAR in the Northern observatory and two Raman LIDARs in the South, all of them validated directly or indirectly (e.g. through the ARCADE project $[49,50])$ by such a LIDAR network. All proposed LIDARs will be operating at $355 \mathrm{~nm}$ and $532 \mathrm{~nm}$ at least, in order to determine the Ångström coefficient in the wavelength range where the CTA telescopes are sensitive to Cherenkov light.

Detailed calculations of the sensitivity of the proposed Raman LIDAR projects have been carried out [51, 52], developed with the aim to reach high precision in the derivation of the aerosol extinction until high altitudes, without introducing observation time loss to the observatory. In this study, the dimmest Raman line will be detected from a distance of $13 \mathrm{~km}$ (i.e. altitude about $15 \mathrm{~km}$ a.s.l., if observed at zenith) with a signal-to-noise ratio of 10 in only one minute. This will permit to fully characterize one lineof-sight a few minutes before and after a CTA observation run, and during the change of Wobble position [53].

Nevertheless, LIDARs cannot scan the FOV observed by the CTA and continuously disturb science observations. Instead, they will be accompanied by a wide-angle telescope, the FRAM [54, 55], designed to produce atmospheric extinction maps through various standard optical filters with high angular resolution and covering a FOV of about $15^{\circ} \times 15^{\circ}$. Maps of integral AOD, each patch determined with better than $3 \%$ precision (see $[55,56]$ for an explanation of the underlying algorithms), are then taken several times during a CTA observation run and interpolated in time. Interlaced Raman LIDAR measurements serve to divide the interpolated AODs in vertical bins and to re-calibrate its integral. These "aerosol extinction hypercubes" (with altitude, wavelength and time as additional dimensions) are then split into a slow component (due to molecular extinction and quasi-stable aerosol layers, like the boundary layer or sometimes even clouds), and a fast one, which changes throughout a science observation run. Historical information from aerosol characterization campaigns [49], and later on the very same database of previous LIDAR/FRAM observations, together with now-cast predictions of the molecular profile from the GDAS or similar, will help to produce the extinction hypercubes and the identification of their components. The expected shifts in CTA performance from a given extinction hypercube are continuously confronted with those of currently used (or foreseen) MC generated Instrument Response Functions (IRF), and a series of systematic errors is calculated and confronted with the CTA requirements. In case one of these systematic errors exceeds the allowed limit, a new "Time Interval" (TI) is communicated to the

\footnotetext{
${ }^{7}$ https://www.earlinet.org/
} 
CTA Software, together with a suitable start time, and an averaged extinction hypercube, to be used for a new MC simulation of the atmosphere. The critical part of this procedure is a robust estimate of the systematic error. For this purpose, MC simulation studies are required to determine the impact of aerosols and clouds at different altitudes, and cirrus clouds covering parts of the FOV of a Cherenkov camera on the angular and energy resolution and bias, if a uniform coverage of the CTA cameras by aerosols has been assumed in the simulations. Previous studies of the typical morphology of clouds and evolution time scales at the CTA sites are needed as input for these simulations [57].

Additionally to that, redundant information is obtained for calibration and cross-checks by photometric measurements of reference stars using a sophisticated, portable small-aperture multi-pixels photon detector, the UVScope and the UVSiPM [58, 59] and the "Cherenkov Transparency Coefficient" (CTC) calculated directly from science data [60].

\section{Intelligent target selection}

Current IACTs effectively lose a considerable amount of their effective duty cycle at the moment when data selection is made offline. This is due to the fact that observation targets use to get chosen according to a fixed schedule and regardless of atmospheric conditions (as long as observations are not aborted at all). The observations suffer then from non-optimal atmospheric conditions along their lineof-sight, instead of actively avoiding them. However, optimal atmospheric conditions may be decisive for the practicability of low-energy analyses [61], those requiring precision pointing [62], or those requiring maximum sensitivity for monitoring purposes, while other types of observations may be practically unaffected. Selecting data according to these criteria later on offline is hence the worst of all strategies, at least in terms of achievable duty cycle, and if sources with less sensitivity to such non-optimal conditions could have been observed instead.

In order to enhance the effective duty cycle of the CTA, we therefore plan to support the short-term scheduling system with online dynamic scheduling capabilities. This system should either enable observation of sources visible under good atmospheric conditions over those covered by clouds or aerosol layers, or otherwise select sources according to minimal impact on the later offline data selection, given a respective atmosphere. Such an intelligent system shall be able to judge at each moment whether the observation requirements are still met, or whether a different source with less strict requirements should be observed instead.

Preferences of one part of the sky over another might be issued in the case of cirrus clouds which rarely cover the entire sky. In order to avoid inefficient "hole hopping" between clouds, a minimum prediction of the movement of such locally limited clouds is necessary, given historical knowledge and the wind direction and speed at the altitude of a cloud, e.g. from the GDAS or similar. See [57] for first attempts in this direction.
For such pointing forecasts, scanning instruments and All-Sky-Cameras (ASC) [63] are suitable. Active scanning instruments should employ wavelengths which do not interfere with the CTA cameras, such as Ceilometers (operating at $905 \mathrm{~nm}$ or $1064 \mathrm{~nm} \mathrm{[64]).} \mathrm{ASCs} \mathrm{which}$ have been largely used during the CTA site selection process [65], are becoming more and more standard tools for cloud detection at world-class astronomical observatories $[66,67]$. These highly sensitive devices are able to detect even fine cirrus, by comparing detected light fluxes from stars with their catalog values. Exposure times of less than one minute are possible, and provide almost contemporaneous cloud maps. Only cloud height cannot be measured accurately, a task left to the ceilometers.

The scenario is then designed in the following way: the ASC takes a picture of the full sky and converts the result in an atmospheric extinction map (AEM). A cloud recognition program applies a threshold to the AEM and decomposes the result in ellipses, yielding the ASC Clouds List (CL). A global offset (e.g. ground layer enhancement) might be part of the decomposed picture and form the first ellipse extending over the complete FOV of the ASC. If available, the knowledge from available Raman LIDAR shots may be included in the decomposition. The Ceilometer receives the full list of possible observation targets in the short-term scheduler and picks those which are close enough to a cloud in the CL as to get possibly affected by it. After discarding those clouds that remain orphan of associated CTA observation targets, the Reduced ASC Clouds List (RCL) is obtained. The Ceilometer points to the center of each cloud in the RCL and takes an extinction profile ${ }^{8}$. The mean altitude of each cloud is added to the RCL. In the case of more than one layer, only the lower one is picked. A now-cast of wind speed and direction at the relevant cloud altitudes is obtained from GDAS and added to the RCL. Using the RCL and historical knowledge, each cloud in the RCL gets propagated in time for a duration corresponding to next scheduling block, and a probability is calculated for each associated CTA observation target to get covered by the cloud. In case the probability lies above a certain threshold, the expected observation parameters for the next scheduling block: reduction of accessible energy range, degradation of energy and pointing resolution and effective areas are calculated. The list of targets with associated cloud coverage probabilities and performance degradation parameters gets returned to the CTA scheduler for evaluation, before the next scheduling block is executed.

\section{Conclusions}

While two years ago an atmospheric calibration strategy for the CTA was still being designed [68], we are now constructing and implementing a clear strategy for such a task. Each part of the mentioned ingredients: molecular density profiles, molecular absorption, aerosol extinction, but also systematic differences between MC simula-

\footnotetext{
${ }^{8}$ This way, it can be always ensured that a Ceilometer shot does not disturb observations from close-by optical telescopes, sensitive to the Ceilometer wavelength.
} 
tion codes $[69,70]$ and those allowed by simplifications in advantage of simulation speed, is required to contribute by less than $2-3 \%$ to the overall uncertainty budget, limited in total to about $7-8 \%$

With these choices, the CTA will provide much more accurate source spectra (including spectral features), light curves and source morphology measurements than its predecessors and enable the start of the VHE precision gamma-ray astronomy era.

\section{References}

[1] Actis, M. et al. Design concepts for the Cherenkov Telescope Array CTA: an advanced facility for groundbased high-energy gamma-ray astronomy, Exp. Astron. 32193 (2011)

[2] Wakely S. P. \& Horan D. TeVCat: An online catalog for Very High Energy Gamma-Ray Astronomy, Proc. $30^{\text {th }}$ ICRC 31341 (2008) http://tevcat.uchicago.edu/

[3] Bastieri D. et al. Energy Calibration of Cherenkov Telescopes using GLAST Data, Proc. 30 ${ }^{\text {th }}$ ICRC 31555 (2008)

[4] Meyer M. et al. The Crab Nebula as a standard candle in very high-energy astrophysics, A\&A 523 A2 (2010), arxiv: 1008.4524

[5] Aleksić J. et al. The major upgrade of the MAGIC telescopes, Part II: A performance study using observations of the Crab Nebula, Astrop. Phys. 7276 (2016), arXiv:1409.5594.

[6] Aharonian F. et al. Observations of the Crab nebula with HESS, A\&A 457899 (2006)

[7] Gaug M. et al. Calibration strategies for the Cherenkov Telescope Array, Proc. SPIE Astron. Tel. + Instr. \#9149-45 (2014)

[8] Bernlöhr K. Impact of atmospheric parameters on the atmospheric Cherenkov technique, Astrop. Phys. 12 255 (2000) astro-ph/9908093

[9] Garrido D. et al. Atmospheric Aerosols at the MAGIC Site, Proc. 33 ${ }^{\text {rd }}$ ICRC 0465 (2013) arXiv:1308.0473

[10] Nolan S. J. et al. Detailed studies of atmospheric calibration in imaging Cherenkov astronomy, Astrop. Phys. 34304 (2010) arXiv:1009.0517

[11] Sobczyńska D.\& Bednarek W. The impact of clouds on image parameters in IACT at very high energies, Proc. $2^{\text {nd }}$ AtmoHEAD Conf., EPJ 8903009 (2015)

[12] Fruck C.\& Gaug M. Atmospheric monitoring in MAGIC and data corrections, Proc. $2^{\text {nd }}$ AtmoHEAD Conf., EPJ 8902003 (2015)

[13] de los Reyes R. Atmospheric monitoring in H.E.S.S., these proceedings

[14] Hahn J. \& de los Reyes R. Atmospheric Monitoring in H.E.S.S., Proc. $2^{\text {nd }}$ AtmoHEAD conf., EPJ 8902002 (2015)

[15] Hildebrand et al. FACT - Measuring Atmospheric Conditions with Imaging Air Cherenkov Telescopes, Proc. $33^{\text {rd }}$ ICRC 0709 (2013)

[16] Dorner D. et al. A method to correct IACT data for atmospheric absorption due to the Saharan Air Layer, A\&A 493721 (2009) arXiv:0808.0279v2
[17] Fruck C. A novel LIDAR-based Atmospheric Calibration Method for Improving the Data Analysis of $M A G I C$, Proc. $33^{\text {rd }}$ ICRC 1054 (2013) arXiv:1403.3591

[18] Font L. Monitoring and Calibration of the Atmosphere in MAGIC, Proc. $33^{\text {rd }}$ ICRC 0090 (2013)

[19] Fruck C. The Galactic Center Resolved with MAGIC and a new technique for Atmospheric Calibration, $\mathrm{PhD}$ Thesis, TU München (2015), https://mediatum.ub.tum. de/node id= 1237928

[20] Acharya B. S. et al. Seeing the High-Energy Universe with the Cherenkov Telescope Array - The Science Explored with the CTA, Astrop. Phys. 433 (2013)

[21] Aleksić J. et al. Optimized dark matter searches in deep observations of Segue 1 with MAGIC, JCAP 28 (2014) arXiv:1312.1535

[22] Aleksić J. et al. Measurement of the Crab Nebula spectrum over three decades in energy with the MAGIC telescopes J. HE Astroph. 530 (2015) arxiv:1406.6892

[23] Abramowski A. et al. Measurement of the extragalactic background light imprint on the spectra of the brightest blazars observed with H.E.S.S. A\&A 550 A4 (2014) arXiv:1212.3409

[24] García-Gil A. et al. Atmosphere Extinction at the ORM on La Palma: A 20 yr Statistical Database Gathered at the Carlsberg Meridian Telescope PASP 122 1109 (2010)

[25] Stull R. B. An Introduction to Boundary Layer Meteorology, Kluwer Acad. Publ. Dordrecht (1988)

[26] Elterman L. Parameters for Attenuation in the Atmospheric Windows for Fifteen Wavelengths, Appl. Opt. 6 745 (1964)

[27] Gaug M. Site characterization for $C T A-N$, these proceedings

[28] Yu H. et al. The fertilizing role of African dust in the Amazon rainforest: A first multiyear assessment based on data from Cloud-Aerosol Lidar and Infrared Pathfinder Satellite Observations, Geoph. Res. Lett. 42 (6) 1984 (2015)

[29] Pachauri R. K. \& Meyer L. A. (eds.) IPCC 2014: Climate Change 2014: Synthesis Report. Contribution of Working Groups I, II and III to the Fifth Assessment Report of the Intergovernmental Panel on Climate Change IPCC Geneva, Switzerland (2014)

[30] Dubovik O. et al. Application of spheroid models to account for aerosol particle nonsphericity in remote sensing of desert dust, J. Geoph. Res. 111 D11208 (2006)

[31] Kandler K. et al. Chemical composition and complex refractive index of Saharan Mineral Dust at Izaña, Tenerife (Spain) derived by electron microscopy, Atm. Env. 41 (37) 8058 (2007)

[32] Levoni C. et al. Atmospheric aerosol optical properties: a database of radiative characteristics for different components and classes, Appl. Opt. 368031 (1997)

[33] Müller D et al. Aerosol-type-dependent lidar ratios observed with Raman lidar, J. Geoph. Res. 112 D16202 (2007) 
[34] Ansmann A. et al. Independent measurement of extinction and backscatter profiles in cirrus clouds by using a combined Ramanelastic-backscatter lidar, Appl. Opt. 31 (33) 7113 (1992)

[35] Andrews E. et al. Climatology of aerosol radiative properties in the free troposphere Atm. Res. 102 (4) 365 (2011)

[36] Haffke M. Atmosphere Is MAGIC. Berechnung und Implementierung neuer Atmospharenmodelle in die MAGIC-Monte-Karlo-Kette, Dipl. thesis Univ. Dortmund, Germany (2007)

[37] Marín J. et al. Density profile characterization and modeling at Paranal and Armazones $2 k$ sites, these proceedings

[38] Vrastil M. Overview of Atmospheric Simulation Efforts in CTA, these proceedings

[39] Daniel M. Application of radiosonde data to VERITAS simulations, Proc 30 ${ }^{\text {th }}$ ICRC 31329 (2008)

[40] Lakićević M. et al. Atmospheric conditions at Cerro Armazones derived from astronomical data, A\&A $\mathbf{5 8 8}$ A32 (2016)

[41] Thompson A. M. et al. Southern Hemisphere Additional Ozonesondes (SHADOZ) 1998-2004 tropical ozone climatology: 3. Instrumentation, station-tostation variability, and evaluation with simulated fight profiles, J. Geoph. Res.: Atm. 112 D03304 (2007)

[42] Cuevas E. et al. Surface O3 characterization in the sub- tropical North Atlantic troposphere, Atm. Chem. Phys. 131973 (2013)

[43] Haynes P. H. \& McIntyre M. E. On the conservation and impermeability therorems of potential vorticity, $\mathrm{J}$. Atm. Sci. 472021 (1990)

[44] Rondanelli R. et al. Rapid changes in ozone mixing ratios at Cerro Tololo $\left(30^{\circ} 10^{\prime} \mathrm{S}, 70^{\circ} 48^{\prime} \mathrm{W}, 2200 \mathrm{~m}\right)$ in connection with cutoff lows and deep troughs, J. Geoph. Res.: Atm. 107 (D23) ACL 6-1 (2002)

[45] Anet J. G et al. Surface ozone in the southern hemisphere: 20 years of data from a site with a unique setting in El Tololo, Chile, Atm. Chem. Phys. 20161 (2016)

[46] S̆kerlak B. et al. A global climatology of stratosphere-troposphere exchange using the ERA-Interim data set from 1979 to 2011, Atm. Chem. Phys. 14913 (2014)

[47] Neu J. L. et al. Tropospheric ozone variations governed by changes in stratospheric circulation. Nature Geosc. 7 (5) 340 (2014)

[48] Kimeswenger S. et al. The Innsbruck/ESO sky models and telluric correction tools - The possibility of atmospheric monitoring for Čerenkov telescopes Proc. $2^{\text {nd }}$ AtmoHEAD conf., EPJ 8901001 (2015)

[49] Valore L. et al. The ARCADE Raman Lidar system for the Cherenkov Telescope Array, Proc. $34^{\text {th }}$ ICRC (2015) arXiv:1509.02681

[50] Iarlori M. Experience with Raman LIDARs, these proceedings
[51] Doro M. et al. Status and motivation of Raman LIDARs development for the CTA Observatory, Proc. $1^{\text {st }}$ AtmoHEAD Conf. (2014) arXiv:1402.0638

[52] Eizmendi M. IFAE-UAB Raman LIDAR Link Budget and Components, Master Thesis UPC \& UAB (2011) http://upcommons.upc.edu/handle/2099.1/12959

[53] Fomin V. P. et al. New methods of atmospheric Cherenkov imaging for gamma-ray astronomy. I. The false source method, Astrop. Phys. 2137 (1994)

[54] Prouza M. et al. FRAM The Robotic Telescope for the Monitoring of the Wavelength Dependence of the Extinction: Description of Hardware, Data Analysis, and Results, Adv. in Astron. 2010849382 (2010)

[55] Ebr J. Aerosol measurements with FRAM telescopes, these proceedings

[56] Janecek P. CTA FRAM update, these proceedings

[57] Adam J. Allsky Cameras and Cloud Detection, these proceedings

[58] Sottile G. et al. UVSiPM: A light detector instrument based on a SiPM sensor working in single photon counting, Nucl. Phys. B Proc. Supp. 239258 (2013) arxiv:1305.2699

[59] Maccarone M. C. Auxiliary instruments for the absolute calibration of the ASTRI SST-2M prototype for the Cherenkov Telescope Array, Proc. SPIE Astron. Tel. + Instr. \#9149-44 (2014)

[60] Stepanik S. Atmospheric monitoring using Cherenkov Transparency Coefficient, these proceedings

[61] Aleksić J. et al. Phase-resolved energy spectra of the Crab pulsar in the range of 50-400 GeV measured with the MAGIC telescopes, A\&A 540 A69 (2012) arXiv:1109.6124

[62] Acero F. et al. Localizing the VHE $\gamma$-ray source at the Galactic Centre, MNRAS 4021877 (2010) arXiv:0911.1912

[63] Mandát D. All Sky Camera for CTA Site characterization, these proceedings

[64] Münkel C. et al. Retrieval of mixing height and dust concentration with lidar ceilometer, Boundary-Layer Meteorol. 124117 (2007)

[65] Mandát D. et al. All Sky Camera instrument for night sky monitoring, Proc. $1^{\text {St }}$ AtmoHEAD Conf. (2014) arXiv: 1402.4762

[66] Nemiroff R. J. et al. Educational Aspects of the CONCAM Sky Monitoring Project, AAS Abs. 32 \#120.04 (2000)

[67] Smith R. et al. The Tololo All Sky Camera, Sci. Detect. f. Astron., Astr. \& Space Sc. Lib. 300379 (2004)

[68] Doro M. et al. Strategy implementation for the CTA Atmospheric monitoring program, Proc. $2^{\text {nd }}$ AtmoHEAD Conf., EPJ 8902005 (2014)

[69] Heck D. et al. CORSIKA: An Air Shower Simulation Program, Astr. Src Code Libr. ascl:1202.006 (2012)

[70] Kertzman M. P. \& Sembroski G. H. Computer simulation methods for investigating the detection characteristics of TeV air Cherenkov telescopes, NIM A 343 629 (1994) 